\title{
Techos de cristal en la Cruzada Nacional y capital social
}

\section{Glass ceilings in the National Crusade and social capital}

\author{
Verónica Martínez Flores ${ }^{a}$, Nemesio Castillo Viveros ${ }^{a}$
}

\begin{abstract}
Resumen
En este artículo de investigación se analiza la implementación de la Cruzada Nacional contra el Hambre en Ciudad Juárez, Chihuahua, México y se pone especial énfasis en la etapa de conformación y consolidación de los Comités Comunitarios. Se plantea que el objetivo de incorporar a las comunidades para atender el problema de alimentación e incidir en el capital social y la cohesión no se logró debido a la falta de respuesta a las demandas hechas por los Comités Comunitarios y de continuidad del programa, lo que repercutió negativamente generando desconfianza hacia las instituciones y entre los miembros de las comunidades, mermando así el capital social. No obstante, se reconoce que las experiencias organizativas de las comunidades se volvieron un insumo para lograrlo y fueron utilizadas como parte de la metodología de la $\mathrm{CNCH}$ durante la elaboración del diagnóstico comunitario y los planes de desarrollo implementados.
\end{abstract}

Palabras clave: política social, Cruzada Nacional Contra el Hambre, capital social, comités comunitarios.

\begin{abstract}
In this research article, the implementation of the National Crusade against Hunger (NCAH) in Ciudad Juarez, Chihuahua, Mexico is analyzed and special emphasis is placed on the formation and consolidation phase of the Community Committees. It is posited that the objective of incorporating communities into the Crusade to attend the problem of food and affect social capital and cohesion was not achieved due to the lack of response to the demands made by the Community Committees and the continuity of the program, which negatively affected it, generating distrust towards the institutions and between the members of the communities, thus diminishing the social capital. However, it is recognized that the organizational experiences of the communities became an input to achieve this and were used as part of the NCAH methodology during the elaboration of the community diagnosis and the development plans implemented.
\end{abstract}

Keywords: social policy, National Crusade against Hunger, social capital, community committees.
Kera Yvoty: reflexiones sobre la cuestión social. Vol. 2, 2017, 84-99.

ISSN (impreso): 2519-7797

a Universidad Autónoma de Ciudad Juárez, Instituto de Ciencias Sociales y. Administración, México.

Correspondencia a: veronica.martinez@uacj.mx

Cita:

Martínez Flores, V. \& Castillo

Viveros, N. (2017). Techos de cristal en la Cruzada

Nacional contra el Hambre.

Las posibilidades del capital social en una política social. Kera Yvoty: reflexiones sobre la cuestión social, 2, 28-43.

Recibido:

o1 agosto 2017

Aceptado:

20 diciembre 2017 


\section{Introducción}

La política social tradicionalmente busca mejorar el bienestar de la población (Jusidman, 2008); en el caso de la política de desarrollo social en México "tiene como objetivo central hacer efectivo el ejercicio y disfrute de los derechos sociales" (PSDS, 2013-2018, p. 23). La Política Social de Nueva Generación (PSNG), como la denomina la administración federal 20132018, busca "superar la pobreza y eliminar las brechas de desigualdad" (p. 43); dando prioridad a acciones como: el desarrollo de capacidades, el acceso a esquemas de seguridad social, la construcción de un entorno digno, la mejora de ingresos, la participación y la coordinación entre gobierno y población. Bajo esta lógica, el bienestar de una población no es producto sólo de las acciones unilaterales del Gobierno Federal, sino de la participación de otros agentes de diferentes órdenes de gobierno y grupos de la sociedad, entre ellos los propios beneficiarios de los programas sociales. Por esto, en el Programa Sectorial de Desarrollo Social 2013-2018 se anuncia como objetivo 5 el fortalecimiento de la participación social como mecanismo para impulsar el desarrollo comunitario; se trata de fortalecer a actores sociales que promuevan el desarrollo de la cohesión y el incrementodel capital social para consolidar mecanismos de participación social donde la ciudadanía sea un sujeto activo. Entre las líneas de acción se consideran la formación de Comités Comunitarios y el fomento de la organización.

Como parte de la PSNG, en enero de 2013 se creó la Cruzada Nacional contra el Hambre $(\mathrm{CNCH})$ la cual se define como una estrategia de inclusión y bienestar social, donde los procesos participativos y el esfuerzo conjunto de los diferentes órdenes de gobierno, así como de otros sectores sociales permitirán el alcance de sus objetivos (DOF, 2013); al año siguiente la estrategia se convertirá en el Programa Nacional México Sin Hambre (DOF, 2014).
Dentro de ella, los procesos participativos fueron diversos, resaltando particularmente la participación social de los grupos comunitarios. Entre 2013 y 2015 estudiantes de diversas universidades de México fueron contratados para impulsar la organización y la participación social a través de la creación de Comités Comunitarios. Su fin último era lograr un diagnóstico y plan comunitario que fueran el sustento para las acciones a seguir por parte de los diferentes órdenes de gobierno; esto a través de un ejercicio participativo. De esta manera, los Comités Comunitarios fueron el principal esquema de participación social dentro de la $\mathrm{CNCH}^{1}$ para incidir sobre la cohesión y el capital social.

En el caso de Ciudad Juárez, se crearon mil 289 Comités Comunitarios en esos años, impulsados por estudiantes y egresados de la Universidad Autónoma de Ciudad Juárez (Martínez Flores \& Romo Aguilar, 2016). En esta ciudad de la frontera de México los esquemas de organización comunitaria no son una herramienta nueva de participación y ya diferentes autores han registrado estas experiencias (Álvarez et al., 2015; Arzaluz \& Jurado, 2006). Desde los años ochenta surgieron organizaciones sociales preocupadas por colaborar en la atención y solución de los problemas más apremiantes que afectaban al país. $\mathrm{Si}$ bien muchas de estas agrupaciones tenían antecedentes en los movimientos populares de los años sesenta $y$, en algunos casos, en movimientos sociales de obreros, campesinos y del magisterio, con trayectorias más largas de lucha, su desarrollo y consolidación fue posible en el contexto de las reformas del Estado implementadas durante la década de los ochenta. En este contexto, la incorporación de la participación de la ciudadanía en

\footnotetext{
1 El Sistema Nacional para la Cruzada contra el Hambre (SNCH) se compone de diversos comités, consejos y comisiones (ver DOF, 2013 y 2014).
} 
los planes y programas de gobierno es un requisito. En el ámbito de la política social y de combate a la pobreza, pueden observarse esquemas de participación social y comunitaria en programas emblemáticos como el Programa Nacional Solidaridad (PRONASOL), Programa de Educación, Salud y Alimentación (PROGRESA) y el Programa Oportunidades. En la actualidad, la estrategia $\mathrm{CNCH}$ incorpora la participación social y comunitaria para la erradicación del hambre y tiene como punto de partida la construcción de la organización y la construcción de capital social.

Este trabajo tiene como objetivo reconocer los elementos de capital social presentes en los Comités Comunitarios de la Cruzada Nacional contra el Hambre en Ciudad Juárez, Chihuahua, y su capacidad para lograr el alcance de los derechos sociales que se establecen en la misma. Este artículo consta de los siguientes apartados: primero se describe de manera general la $\mathrm{CNCH}$, posteriormente se desarrollan los principales acercamientos a la teoría de capital social; luego se da cuenta de los hallazgos; y cierra con algunas conclusiones.

\section{Cruzada Nacional Contra el Hambre}

La Cruzada Nacional Contra el Hambre se creó por decreto presidencial el 22 de enero de 2013; en ella se "reconoce que la privación de alimentos es producto de un entorno socioeconómico complejo, multidimensional, que requiere de un enfoque de carácter integral que involucra múltiples instrumentos de política pública en materia de alimentación, salud, educación, vivienda, servicios en la vivienda e ingresos" (Sedesol, 2017, s.p.); parte de la idea de un proceso participativo donde se conjunta el trabajo de los tres órdenes de gobierno, la sociedad y la ciudadanía en general a través del Sistema Nacional para la Cruzada Nacional contra el Hambre que se compone por el Consejo de Expertos de la Cruzada contra el Hambre, la Comisión Intersecretarial para la Instrumentación de la $\mathrm{CNCH}$, el Consejo Consultivo de $\mathrm{CNCH}(\mathrm{CCNCH})$, los Comités Estatales Intersecretariales de la $\mathrm{CNCH}$, Comisiones Intersecretariales Municipales y los Comités Comunitarios (DOF, 2014). Entre sus objetivos se encuentran:

i. Cero hambre a partir de una alimentación y nutrición adecuada de las personas en pobreza multidimensional extrema y carencia de acceso a la alimentación.

ii. Disminuir la desnutrición infantil aguda y crónica, y mejorar los indicadores de peso y talla de la niñez.

iii. Aumentar la producción de alimentos y el ingreso de los campesinos y pequeños productores agrícolas.

iv. Minimizar las pérdidas postcosecha y de alimentos durante su almacenamiento, transporte, distribución y comercialización.

v. Promover el desarrollo económico y el empleo de las zonas de mayor concentración de pobreza extrema de alimentación.

vi. Promover la participación comunitaria para la erradicación del hambre (DOF, 2014, s.p.)

Cabe destacar que el programa pretende ser de largo alcance, en el sentido de que busca generar "la autogestión y el capital social de la propia comunidad,y partedel empoderamiento de las personas para que puedan constituirse en protagonistas de su propio desarrollo" (Sedesol, 2017, s.p.). La participación social a través de los Comités Comunitarios es entonces central en el programa, ya que es a partir de ella que pueden identificarselasnecesidadesdelascomunidades y priorizarse las acciones (Sedesol, 2017) que den como resultado la creación de capital social y bases para la cohesión social.

Los Comités Comunitarios
son el mecanismo de participación social integrados por miembros de la comunidad que de alguna manera son receptores de diferentes programas 
sociales y se constituyen como el órgano de representación de la comunidad; se instituyen por medio de una asamblea comunitaria y se formalizan a través del Acta Constitutiva de los Comités Comunitarios; cuentan con una Mesa Directiva que se compone de Presidente, Secretario, Tesorero y vocales de comisiones diversas (Sedesol, 2013).

Estos comités tienen entre sus responsabilidades la elaboración de un diagnóstico y de un plan comunitario. El plan comunitario es el resultado del trabajo realizado por los Comités Comunitarios y el acompañamiento de un(a) brigadista comunitario (a); quienes a través de una metodología participativa, que contempla una serie de asambleas, desarrollan un diagnóstico, priorizan necesidades $y$ acuerdan acciones.

De acuerdo con el Gobierno de la República (s.f.), se instalaron 72 mil $082^{2}$ Comités Comunitarios en 911 municipios a nivel nacional; durante 2014 se realizaron 70 mil 699 planes de desarrollo comunitario con la colaboración de 8 mil 494 brigadistas 3 , fortaleciendo con estas acciones el capital y la cohesión social. Para 2017, la página de Sin Hambre reporta 8 mil o77 brigadistas y mil 12 municipios atendidos ${ }^{4}$.

\section{Capital Social}

En los estudios que involucran el concepto de capital social no se encuentra hasta la fecha una definición que resulte completamente satisfactoria para la comunidad científica. Dicho de otra forma, no existe una definición clara sobre lo que implica hablar de capital social, sin embargo, hay algunos acercamientos que pueden resultar útiles para situar su

2 En 2013 se instalaron 57,185 y 14,897 en 2014 (Gobierno de la República, s.f.).

3 En este artículo se utiliza de igual manera brigadista o promotor para dar cuenta del personal que promovía la conformación de los CC y daba seguimiento a los mismos dentro de la $\mathrm{CNCH}$.

4 http://sinhambre.gob.mx/mapa/ revisado el 24 de julio de 2017. No se reporta la fecha de actualización. definición desde la óptica social. Pierre Bourdieu publica en 1980 un artículo de investigación donde define el capital social como "recursos potenciales $o$ actuales asociados a la posesión de una red duradera de relaciones más o menos institucionalizadas de conocimiento y reconocimiento mutuo. Expresado de otra forma, se trata aquí de la totalidad de recursos basados en la pertenencia a un grupo. El capital total que poseen los miembros individuales del grupo les sirve a todos. En la práctica, las relaciones decapital social sólo pueden existir sobre la base de relaciones de intercambio de materiales y/o simbólicas, y contribuyen además a su mantenimiento" (Bourdieu, 1986, p. 249). De esta manera, Bourdieu permite pensar en la posibilidad de construcción de capital social en las comunidades, en donde cada integrante puede introducir conocimiento, experiencias o capacidades para enriquecer la facultad creativa de los grupos sociales, destacando el hecho de que ellos mismos la pueden innovar, para favorecer los procesos de su propio desarrollo.

Putnam (1993) entiende por capital social un conjunto de redes sociales que crean un cierto nivel de vida asociativa en una comunidad determinada y en cuyo seno se generan normas de reciprocidad donde la confianza interpersonal es la clave que posibilita la cooperación y facilita acciones coordinadas que dan paso a la eficiencia social. En suma, puede colegirse que el capital social está definido en función de la densidad asociativa de una sociedad específica.

En Making Democracy Work, Putnam observa que, en principio, la densidad asociativa al interior de las redes sociales permite que los ciudadanos que participan en asociaciones voluntarias y esencialmente horizontales (desde clubes de fútbol hasta grupos corales o clubes de lectura) planteen de forma agregada demandas que beneficien a la sociedad en su conjunto. Así, revela el estudio hecho para Italia, cuanto mayor sea la densidad de vida asociativa, mayor será la capacidad de los ciudadanos 
de una región determinada para cooperar y actuar para obtener beneficios mutuos $y$, en consecuencia, plantear demandas de bienes públicos y mejorar la respuesta por parte de los políticos regionales. La tesis de Putnam, desde luego, ha sido recibida con interés y escepticismo. Varias reflexiones señalan algunos problemas en la definición de capital social del autor, así como en la relación causal entre capital social y desarrollo institucional-social, debido a la ambigüedad en su definición de las instituciones. No obstante, el libro demuestra la importancia que tiene el capital social para resolver los problemas de comportamiento egoísta, dilema típico de la acción colectiva. Putnam pone en claro que para el desarrollo social y económico el capital social representa una gran ventaja que favorece la calidad de vida de las sociedades, tomando en cuenta que no se espera que el Estado sea el promotor del desarrollo, sino que las propias comunidades apuestan por mejorar sus condiciones.

Por su parte, Coleman identifica el capital social a partir de la estructura social, en el entendido de que ésta "pone a los actores como recursos y que ellos los pueden usar para lograr sus propios intereses. Todas las relaciones sociales y estructuras sociales facilitan algunas formas de capital social; los actores establecen relaciones a propósito y las continúan para seguir proporcionando beneficios. Sin embargo, ciertos tipos de estructura social son especialmente importantes para facilitar algunas formas de capital social" (1988, p. 101-102). El autor confía en el actor social, las redes sociales y la estructura social para generar el capital social; en el caso de las políticas sociales el problema se presenta cuando hay desconfianza, misma que ha sido producida por las propias estructuras institucionales, entonces la generación de nuevo capital social recae en los actores sociales que implementan los programas sociales, quienes tienen que enfrentarse a la apatía de los beneficiarios y la reglas de la administración pública para lograr generar redes sociales que sean la base para la renovación del capital social.

De acuerdo con la CEPAL, el capital social tiene efectos positivos "como control social, creación de confianza entreindividuos, cooperación coordinada, resolución de conflictos, movilización y gestión de recursos comunitarios, legitimación de líderes y generación de ámbitos de trabajo, la prevención y sanción de quienes abusan de él y la producción de bienes públicos" (CEPAL, 2001, p. 4). Regularmente se suele pensar en las ventajas que da el capital social al desarrollo comunitario, o incluso al desarrollo económico. Teóricamente, se acostumbra considerar el capital social como una herramienta que genera confianza y cohesión social en las comunidades; sin embargo, es necesario conocer las formas o prácticas sociales que pueden darse dentro de las comunidades y que pueden ser diferentes a lo que se espera como capital social.

Diversos estudiosos del tema consideran que el capital social puede ser pioneroen lageneración deldesarrollosocial y económico, sin embargo, hay perspectivas que cuestionan esta idea $\mathrm{o}$, al menos, hacen algunas preguntas hacia este precepto. Barbara Arneil pregunta ¿es tan poderoso el capital social? No necesariamente, responde, pues hay diversas comunidades. De hecho, hay una buena razón para pensar que una fijación con capital social es equivocada, incluso peligrosa para grupos menos poderosos de la sociedad, como mujeres, minorías étnicas, gays y lesbianas y personas con discapacidades (Arneil, 2006) debido a los intereses individualistas o al egoísmo. Arneil sostiene que la división, la diversidad y los conflictos deben ser considerados como un desafío en el contexto norteamericano, poniendo especial atención en la dominación de los hombres blancos nacidos en el país y de clase media, lo que dificulta implementar la idea de capital social.

Arriagada destaca el riesgo que puede suponer el capital social, ya que "las redes, 
contactos y asociaciones están al servicio de las actividades ilegales" (2003, p. 572); y retoma a Rubio, quien muestra un contexto donde el capital social no produce efectos positivos: "la existencia en Colombia de una economía ilegal importante y creciente, fomentada por organizaciones criminales de mucho poder, que ha dado origen a una institucionalidad paralela que retribuye y favorece comportamientos criminales. El autor muestra cómo altos niveles de capital social dentro de las organizaciones criminales son reorientados hacia actividades extra-legales que reditúan altos beneficios para aquellos involucrados en estas actividades" (Rubio citado por Arriagada, 2003, p. 572).

El capital social bien orientado puede potenciar la cohesión comunitaria: debido a que las personas confían más entre ellas, se genera mayor comunicación y pueden identificar de manera colectiva los problemas y ayudarse mutuamente, es decir, existen razones por lascuales el capital social puede ser un activo importante para el desarrollo comunitario; sin embargo, implementarlo no es tan sencillo y esperar que seden los resultados comoteóricamente se piensa, no es objetivo porque dentro de las comunidades hay prácticas ancladas en el autoritarismo, clientelismo, el paternalismo, el egoísmo, entre otras, por lo que, al querer implementar el capital social como estrategia de intervención social, estas formas pueden surgir dentro de las comunidades y los objetivos que se habían marcado como positivos, en la práctica pueden ser negativos.

Arriagada dice que el clientelismo es una de las formas de capital social que puede ser negativo, debido a que no se promueve el desarrollo de las comunidades, al contrario, se convierten en receptoras de la política social: "el clientelismo es uno de los problemas centrales y más antiguos en la relación entre organizaciones comunales y de base, y los agentes estatales y no gubernamentales en América Latina. Pueden distinguirse distintos tipos de clientelismo en un continuo que va desde el autoritario al paternalista. A tales tipos de relación pueden agregarse otros que son más proclives a potenciar el capital social colectivo, como la capacitación de los miembros de las organizaciones hasta que éstas alcancen su autonomía, y a quienes los funcionarios públicos y privados deben rendir cuentas" (2003, pp. 572-573).

Una comunidad que se organiza para linchar a un violador o asesino o los grupos que se organizan para fines del narcotráfico son ejemplos de capital social negativo. Estos grupos se crean de manera colateral y su fin no favorece el Estado de Derecho, es decir, un lugar donde se cumplan las leyes. De acuerdo con Rivera, este tipo de capital social se produce en grupos muy particulares y también son organizaciones "construidas y mantenidas con base en fuertes lazos informales como la confianza, la reciprocidad y la lealtad, que, aunque pueden rendir altos beneficios a sus miembros, asimismo pueden ser negativos y altamente dañinos para el entramado social en el que se desarrollan" (2016, p. 118). De manera esquemática se muestra en la Tabla 1 estas distinciones.

El capital social positivo puede generar confianza entre vecinos, diálogo y comunicación o trabajo colectivo para resolver problemas comunes. El capital social negativo permite la creación de redes sociales delictivas, conflictos entre vecinos por intereses distintos o el uso de trabajo colectivo para favorecer a un partido político o intereses de líderes.

En resumen, se observa que el capital social da posibilidades de creación de confianza, cooperación, resolución de conflictos, movilización y gestión de recursos comunitarios, legitimación de líderes y generación de ámbitos de trabajo, sin embargo, en algunas circunstancias estos recursos pueden también ser usados para actividades en contra de la armonía social.

\section{Enfoque metodológico}

La investigación de corte cualitativo 
ofrece una visión complementaria, con hallazgos no generalizables sino aproximaciones a las diversas problemáticas a partir de las miradas de los sujetos sociales, por lo que demanda un esfuerzo sistemático y un riguroso diseño teórico y metodológico. El enfoque cualitativo pone especial énfasis en la valoración de lo subjetivo y vivencial y en la interacción con el sujeto; privilegia lo local, lo cotidiano y lo cultural para comprender la lógica y el significado que tienen los procesos sociales para los propios actores, que son quienes viven y producen la realidad sociocultural. El enfoque cualitativo tiene una perspectiva holística que empuja al investigador a valorar escenarios, personas, contextos, grupos y organizaciones como un todo no reducible a variables. Desde este punto de vista, los fenómenos se estudian desde una visión historicista, desde el contexto de su pasado y en el de las situaciones actuales, entendiendo que el presente contiene en germinación aspectos del futuro (Galeano, 2007).

Esta investigación sigue los citados postulados, por lo que es de tipo descriptivo y exploratorio. Lo descriptivo implica que se busca únicamente conocer las situaciones o acontecimientos sociales; no existe interés en comprobar explicaciones, ni en probar hipótesis, ni en hacer predicciones. Por otro lado, es un estudio exploratorio porque se recogen e identifican antecedentes generales, temas, teorías y conceptos que hacen referencia al problema planteado. Por lo tanto, no se pretende generalizar los resultados ni los hallazgos encontrados en el curso de la investigación.

Para este trabajo, se usó la entrevista como técnica de recolección de información; herramienta cualitativa de gran utilidad en la medida en que se fundamenta en la interrelación y la comunicación humana, ya que "la entrevista proporciona un excelente instrumento heurístico para combinar los enfoques prácticos, analíticos e interpretativos implícitos en todo proceso de comunicar" (Sierra, 1998, p. 277). En sentido teórico, la entrevista es un intercambio verbal que permite reunir información de carácter privado y cordial durante el proceso de investigación, donde una persona cuenta su historia, la versión subjetiva de los hechos y responde a preguntas, de acuerdo con la grande o pequeña parte que le tocó vivir, en relación con un problema y/o fenómeno en específico; es pues una comunicación interpersonal para la obtención de información (Sierra, 1998).

\section{Experiencias comunitarias de organización: confianza}

Es importante reconocer que cuando un programa social interviene en una comunidad, ésta no es una tabla rasa, sino que trae consigo experiencias de participación previa, logros y fracasos, relaciones de colaboración o de conflicto. El caso de las comunidades participantes en los Comités Comunitarios no fue la excepción, en las entrevistas realizadas a las presidentas de éstos, todas ellas hacían referencia a experiencias de organización

Tabla 1

Tipología del capital social

Capital social negativo

Efectos

Capital social positivo
Creación de redes sociales delictivas.

Conflictos entre vecinos por intereses distintos.

Uso de trabajo colectivo para favorecer a un partido político o intereses de líderes.
Generación de confianza entre vecinos.

Creación de diálogo y comunicación entre vecinos.

Trabajo colectivo para resolver problemas comunes.

Fuente: Elaboración propia. 
que les permitieron ir modificando su contexto inmediato:

\begin{abstract}
Ahorita estamos contentos porque duramos muchos años gestionando la carretera de enseguida, aquí la de la pagos. Fueron años de gestionarla y pues ya la gente estaba desilusionada ¿verdad? Iba yo y me decían: "Bueno, pues bueno, quieres hacerle la lucha". Y ahorita la gente está muy contenta porque nos están haciendo una avenida muy bonita. (Entrevista personal RS, Ciudad Juárez, 2015)
\end{abstract}

La bibliografía sobre el tema ha demostrado que en las colonias que tienen un origen popular por lo regular existe la organización y la cooperación entre los habitantes, pues se requiere del trabajo colectivo para gestionar recursos que mejoren las condiciones de vida de las familias. Lo que queda claro en los datos analizados es que, de manera colectiva y sin necesidad de la intervención del gobierno, las comunidades han logrado ponerse de acuerdo para satisfacer sus necesidades:

Pues no había pavimento aquí, ni aquí ni acá, pues varia gente estaba... el rebote era la escuela, ahí era la primaria. El cuarto que se está utilizando como dispensario médico ahí ahorita, era la primaria; dos cuartitos era la primaria. Como en el 76 se compraron el terreno los mismos colonos de aquí -mi papá era uno de ellos- muy activos. Compraron el terreno, se compraron... compraron el terreno para hacer la primaria ahí abajo, y él fue el que apoyó mucho en la primaria, para hacer la primaria. (Entrevista personal CR, Ciudad Juárez, 2015)

Se observa que las comunidades han sido gestoras de su propio desarrollo. No obstante que algunos gobiernos las conciben como infantes se puede observar que sus experiencias organizativas son muy importantes, pues les permiten hacer frente a los problemas que se les presentan, crear redes de apoyo mutuo y generar confianza entre sus miembros:

$$
\begin{aligned}
& \text { Sí, sí, sí: necesitamos algo y vamos } \\
& \text { con fulano [para que] nos ayude. } \\
& \text { (¿Entonces me decía, sí hay como } \\
& \text { confianza en la comunidad para } \\
& \text { ayudarse?) Sí, sí hay para que cuando } \\
& \text { se necesite algo se ayuda, se ayuda a } \\
& \text { esa persona, sí. (Entrevista personal } \\
& \text { CR, Ciudad Juárez, 2015) }
\end{aligned}
$$

Si bien es cierto que elementos de capital social positivo como la confianza y las redes de apoyo se presentan en las comunidades; también lo es que se encuentra capital social negativo: en este caso se reconocen prácticas clientelares y paternalistas heredadas de años de acciones sociales condicionadas a lógicas partidistas. Es decir, hay interés en participar siempre y cuando se ofrezca algo a cambio; esto es uno de los problemas que se presentaron en la conformación de los Comités Comunitarios en Ciudad Juárez: una participación que esperaba ser estimulada con "ayudas" por parte de los implementadores de los programas sociales:

[La promotora me dice] que pase con los vecinos a invitarlos para formar el Comité de Cruzada sin Hambre. Vamos, los invito casa por casa: duros de convencer, como le digo, porque pues son puras promesas prácticamente, la verdad. Entonces logran venir 30 o 40 gentes, sí logran venir; a veces que hasta más gente porque la gente aquí alrededor sabe queva a haber ayudasyacuden, pero se desaniman cuando ven que ya no pasa nada, no pasa nada más que la junta esa inicial y ya. (Entrevista personal CR, Ciudad Juárez, 2015)

En la descripción anterior se refleja 
que hay interés en la participación y en la organización a partir de la existencia de lazos de confianza construidos previamente por la presidenta como líder de la colonia, pero que ésta es difícil de mantener cuando no existen resultados tangibles. Se observa que el personal que promovía los Comités Comunitarios de la $\mathrm{CNCH}$ utilizó el capital social existente en las comunidades intervenidas para impulsar su propuesta y, particularmente, la participación:

Pues sí, ajá, sí me gustaba. Sí me gustaba y más que nada porque la gente estabaal pendientedela colonia, de, como vecinos: "Miren, sabe que ya venía. Mire, y esta señora no tiene esto. Mira, esta señora tiene... No, pues vamos a exponerlo". O sea, como comunidad nos involucramos mucho para nuestra colonia. Entonces eso me gustaba, esos comités me gustaban mucho. Y yo les hablaré: vénganse. Pero, pues, cuando ya el momento que ya no vinieron pues ya no tuve qué decirles, pues qué les decía si ellos venían. Y me dice: ¿Sabes qué? Así y así. Cuando tenga la oportunidad pues yo se los digo, pero me gustaría más que se involucraran junto con nosotros. (Entrevista personal YS, Ciudad Juárez, 2015)

Como se aprecia, la experiencia de organización de las comunidades intervenidas dentro de la $\mathrm{CNCH}$ en Ciudad Juárez fue un insumo importante para lograr la participación en los Comités Comunitarios. Así, la confianza y las redes de apoyo construidas históricamente se vuelven recursos de los cuales pueden echar mano los promotores de una política social con enfoque participativo como la que se estudia; sin embargo, como ya se ha señalado, este capital social no siempre es positivo; en ocasiones ciertas prácticas han resultado en capital social negativo, como una relación clientelar, convirtiéndose en un peligro para el impulso de los derechos sociales y la cohesión social.

\section{Diagnósticos comunitarios y la generación de capital social}

Una de las estrategias que ha generado resultados en capital social es la organización social. La CNCH recurrió a la organización de las comunidades mediante el esquema de asambleas comunitarias para aglutinar a la comunidad y trabajar con ella en la identificación de sus necesidades y la discusión de los problemas de su comunidad con el interés de que el Estado coadyuvara en su resolución:

Hacíamos las asambleas para seguir este... gestionando los problemas, los problemas de la comunidad, sobre todo las gestiones que necesita la comunidad. Y de hecho todavía se siguen haciendo esas asambleas, aquí conmigo cada viernes se hacen reuniones. Aquí tenemos reuniones cada viernes, el comité completo; cada viernes tenemos reuniones, está el comité completo y aparte toda la comunidad viene a ver qué es lo que sigue, qué es lo que vamos a exponer. De hecho, aquí con nosotros no se han dejado de hacer reuniones. (¿Lo siguen haciendo cada viernes?) Seguimos haciéndolo cada viernes (¿Y que trabajan cada viernes?) Cada viernes vemos de lo que viene, de los trabajos, de la necesidad de los jóvenes. Más que nada ¿verdad? nos importa que [en] la comunidad haya trabajo para los jóvenes, bajar los programas temporales que vienen, más que nada tenerlos nosotros para la comunidad y todo eso. Es lo que nos está apoyando. Y luego saber quién es la gente que falta, que no está en la Cruzada contra el Hambre, saber quién necesita del programa. Todo eso de lo que nosotros gestionamos aparte. (Entrevista personal RS, Ciudad Juárez, 2015)

De esta manera, las asambleas comunitarias se establecieron como un 
espacio para el diálogo y la construcción de consensos. A partir de ellas y de la organización generada se fue desarrollando el diagnóstico comunitario que permitió a las comunidades reconocer los principales problemas y necesidades que tienen los habitantes; se presentaba como un lugar en el cual las personas discutían de manera colectiva las demandas más relevantes de la comunidad:

Yo estoy participando como presidente de comité de la Cruzada contra el Hambre. Yo me enteré porque vinieron a pedir mi apoyo para que... por los vecinos, para que los apoyara con la comunidad. Entonces este... yo estoy trabajando con los vecinos, a función de que estaban todos ellos. Cada vez que hacemos reuniones exponemos los problemas que tenemos en la comunidad. Entonces ya nosotros se los exponemos a la gente que viene de la Cruzada contra el Hambre. Entonces ya ellos nos empiezan a apoyar. (Entrevista personal RS, Ciudad Juárez, 2015)

Las asambleas eran un momento para la interlocución entre promotor(a) y los miembros de la comunidad, donde las necesidades e inquietudes de los individuos eran expuestasantequien, para losasistentes, era la representación del Gobierno y quien recolectaba la información:

Venía la muchacha y le dábamos señas de... la muchacha pues nos juntaba y nos veníamos, y cada quien opinaba; o sea cada miembro de la colonia decía: "Oiga, pues yo necesito la ayuda del adulto mayor." "Yo necesito la ayuda de los niños de síndrome de Down." O sea, todo ese tipo de ayuda. La gente venía y se las exponía a la muchacha que venía y ya ella anotaba cada inquietud de cada quien. Y ella es la que iba; decía que ella la exponía allá más para adelante. (Entrevista personal YS, Ciudad Juárez, 2015)

Sin embargo, aunque estas demandas eran tomadas en cuenta por el personal, su capacidad de incidencia para resolverlas era mínima, pues lo que se buscaba con la organización a través de la conformación de los comités de la $\mathrm{CNCH}$ era reconocer los problemas de la comunidad para planear las acciones a realizar mediante el programa y la incorporación de diferentes instituciones para la atención de los problemas, en función de lo priorizado por los propios vecinos.

La organización social, la cooperación, y la confianza permiten que las comunidades seintegrenydiscutan las necesidadessociales que enfrentan en lo cotidiano, estos fueron insumos para la creación de los diagnósticos comunitarios requeridos por la $\mathrm{CNCH}$. En los casos analizados, en su mayoría trabajaron en ello solamente algunos miembros de la mesa directiva, desaprovechando así un recurso participativo importante para la creación de cohesión social. Se contaba con diferentes herramientas para la realización del diagnóstico: recorridos, elaboración de transectos y diversos formatos que tenían que ser llenados por los miembros de la mesa directiva y comisiones de trabajo:

Me dejaban papeles para ver las necesidades de cada casa de la comunidad, de qué pasaba en nuestro alrededor. Preguntas que se les podían hacer aquí a los vecinos, sólo hasta ahí llegaba. Y qué necesidades tienen, qué necesita, qué le falta; y hasta ahí llegaba nada más. (OK ¿Y entonces usted llenó toda esta papelería, usted y el comité o usted sola?) Entre mi secretaria y el presidente y algunos vecinos que me acompañaban para ver si yo hacía lo que estaba haciendo. (¿Y así obtuvieron la información?) Sí, así obtenemos información. (Entrevista personal CR, Ciudad Juárez, 2015) 
Cabe destacar que en realidad los Comités Comunitarios identificaron problemas de carácter público y de competencia municipal, y no tanto en relación con la alimentación o la nutrición de las familias:

Pues en ese tiempo estábamos identificando que el alumbrado, prácticamente pues estamos en todas las calles, dos, tres lámparas prenden y después de cinco minutos prendidas y se apagan, y ya no prenden hasta después de media hora. ¿Qué más? Pues hay casas que están vacías pues que van y las utilizan como nido para drogarse, para hacer cosas y este.... ¿Qué más? Pues que pasara la policía, que pasara constantemente. $Y$ pues sí, últimamente empezó a pasar, pero pues porque nos juntamos varios vecinos que vivíamos ahí, les hablamos y les dijimos y empezaron a pasar constantemente las patrullas; por lo mismo porque había muchas casas vacías; de hecho, todavía hay muchas casas vacías. Y ya. (Entrevista personal CR, Ciudad Juárez, 2015)

La información de carácter participativo generada en el diagnóstico comunitario fue el insumo para la construcción del plan comunitario, el cual debía contener los principales problemas sentidos por los miembros de los comités y de los vecinos para que, en una siguiente etapa de la $\mathrm{CNCH}$, fueran implementados programas para atenderlos de manera coordinada:

Se supone con esta construcción del diagnóstico, le llamaban plan comunitario, se construyeron unos documentos. (¿Usted conoció este documento?) Pues recuerdo mucho que nos tomó unas firmas, de primero fueron una firma aquí para que la gente que estaba, que asistió a la asamblea y que estaba de acuerdo con lo que habíamos acordado en cada una, siempre era tomada de lista, o sea de apuntarse quién asistió, y que si estaban de acuerdo con lo que se había estipulado aquí. (¿A eso le llamaban acta?) El acta de asistencia, y de que todos estaban de acuerdo. Y nosotros cada cosa que se acordaba que se iba a hacer, la firmábamos nosotrosy se entregaba. O sea, eran los programas que nosotros como comité estábamos pidiendo para la colonia: las necesidades. (Entrevista personal YS, Ciudad Juárez, 2015)

Además de los problemasterritoriales, en las comunidades se identificaron las dificultades que viven ciertos grupos de la población, como los adultos mayores y las madres solteras o jefas de familia. Por ejemplo, AL (seudónimo) comentaba que en su comunidad las principales necesidades se dan entre adultos mayores, quienes se encuentran muy vulnerables, además de niños que no tienen quién los cuide porque sus mamás se tienen que ir a trabajar a la maquiladora:

Ay, pues es que hay muchas, muchas personas de la tercera edad que no tienen ninguna ayuda de... ni seguro, ni nada, o sea: de plano están sin nada, sin nada. Este... hay mucho problema de desempleo, en ese tiempo había mucho problema de desempleo, ya gracias a Dios se ha ido quitando. Este... mucha madre soltera que no tenía cómo dejar a sus niños en una parte específica que pudieran ellas ir a trabajar. Pues es lo que más marcado estaba: muchos viejitos solos, viviendo en casas solitos, con sus casas cayéndose. $\mathrm{O}$ sea, sí fue impresionante andar casa por casa y ver las necesidades que tenía cada persona, porque sí son muchas. Pues nosotros nos movimos, anduvieran... anduvimos en el sol y todo, buscando y todo, y yo junté todo y no: nada 
más vino esa persona, los jóvenes de la universidad a recoger la papelería y fue todo lo que hicieron, y ya no han vuelto. (Entrevista personal AL, Ciudad Juárez, 2015)

El diagnóstico comunitario utilizado en la $\mathrm{CNCH}$ es una herramienta adecuada para identificar necesidades y conocer problemas pues permite reconocer éstos en su contexto social y territorial; por ejemplo, el caso de los niños de madres trabajadoras de la maquila es un fenómeno que debe ser explicado a partir de la dinámica económica de Ciudad Juárez, Chihuahua, lo que debería permitir crear esquemas de intervención acordes a estas realidades sociales tomando en cuenta sus particularidades. En este sentido, la construcción de dichos diagnósticos y planes comunitarios debió ser la base para la intervención de los programas, pero en la mayoría de los casos esto no sucedió, por lo que generó desconfianza ante la falta de respuesta puntual a las demandas y la falta de continuidad del trabajo comunitario.

\section{Desconfianza ante falta de continuidad}

De acuerdo con el diseño metodológico de la implementación de los Comités Comunitarios, la identificación de necesidades y su priorización a través del diagnóstico y del plan comunitario eran las bases para iniciar la atención focalizada:

Pues haciendo este comité empezamos los vecinos a gestionar para que se nos apoyara con un parque que teníamos muy descuidado, que es el parque Baeza. Empezamos a gestionar y por parte de los promotores y sobre todo por el promotor A -él era el que siempre nos estuvo apoyando para que nos ayudaran con el parque- y este, y de repente nos llega la noticia de que se nos escuchó y que nos van a remodelar el parque. Y este, nos entrega el parque remodelado bien, bien construido, hechoy todo. Yviene la delegada a entregarnos el parque. (Muy bien, ¿y eso cuándo fue?) Fue... no me acuerdo exactamente, pero fue el año pasado, no me acuerdo exactamente el mes; pero luego, luego nos los entregaron. Nos hicieron caso y luego, luego empezaron a ayudarnos con el parque. (Entrevista personal RS, Ciudad Juárez, 2015)

Si bien esta solicitud tuvo una respuesta positiva y rápida a una demanda concreta de la comunidad, en la mayoría de los casos analizados no la hubo, como tampoco existió continuidad en el trabajo de los comités. La organización social promovida dentro del programa permitió que las personas tuvieran una actitud activa en la identificación de sus necesidades y claridad en las demandas sociales; sin embargo, la continuidad de esta participación dependía de la capacidad de los tres órdenes de gobierno para responder a las mismas; en la mayoría de los casos revisados el proceso iniciado con la interlocución entre Estado y ciudadanía a través de los y las brigadistas se frenó por la falta de solución a los problemas expuestos por las comunidades o, en el mejor de los casos, por las respuestas lentas; acciones que obstruyeron el trabajo y, lo que es más peligroso, generaron desconfianza hacia la institución pública:

Sí, y luego vino el muchacho ya en la tercera, en la tercera reunión ya vino. Se llevó todo ese papeleo, y las firmas y... por ejemplo, las credenciales. Y sí, ya de ahí ya no regresó. Nomás nos dijo que nos iba a quedar de hablar luego para ver... por si había una bajada de... por ejemplo, de ayuda o algo. Pero pues no, ya ve: ya tiene mucho que ya no. Yo por eso le digo que ya ni en cuenta. ¿Sí me entiende? $\mathrm{Ni}$ cuenta de eso de SEDESOL ni nada. Y pues yo dije: no, pues ha de ser como... pues nomás para robarnos 
votaciones o algo así. Pues yo pienso; yo, es lo que pensé, pero quién sabe. (Entrevista personal TP, Ciudad Juárez, 2015)

Ante la pregunta constante de las presidentas de comités sobre la continuidad de éstos, y la respuesta a sus demandas, el personal se disculpaba justificando la falta de continuidad por la época electoral que se avecinaba en el estado; sin embargo, con el paso de los meses los y las brigadistas no volvieron a las comunidades, y las presidentas de los comités tuvieron que hacer frente a los reclamos de los vecinos:

Pues de hecho ellos sí, pues ellos sí... algunos se molestaron. No le digo que casi ya se me querían echar a mí encima porque pensaban que como no llegaba ninguna respuesta y eso, y como yo andaba enfrente de eso, primero que invitándolos, y así y así, y pues ya al último como que se desanimaron y como vieron que no había respuesta sobre.... ni de ayudas, ni nada lo que nos habían prometido pues ellos pensaban -yo creo- todo lo que, por ejemplo, todo lo que, a lo mejor todo lo que llegaba yo me lo quedaba. O no sé. Eso lo que pensaron ellos. Y pues digo yo: ¿Cómo?, si yo tampoco he tenido ningún beneficio, ni nada. Y pues no, en realidad nunca miré nada. Y le digo: yo tampoco; yo me desanimé mucho y dije: no, yo tampoco ya en esos comités yo ya no me vuelvo a meter. Porque son puros problemas en lo que uno se mete en realidad. (¿Sí?, ¿por qué?) Pues por lo que le digo: porque como no hay ninguna respuesta sobre ayudas ni nada, uno queda mal con las personas. (Entrevista personal TP, Ciudad Juárez, 2015)

Deesta manera, loque se pensaba ibaa lograr la cohesión social y el fortalecimiento del capital social en las comunidades, llegó a convertirse en motivo de conflicto que trajo consigo la generación de desconfianza entre los miembros de la comunidad y lo que es peor aún, desconfianza en las líderes, para quienes, en algunos casos, era su primera experiencia de participación, creando en ellas apatía hacia la misma.

En el momento de levantar los datos de esta investigación, la etapa de consolidación de los comités comunitarios había terminado. Sólo en dos casos de los seis revisados se mantenía el comité comunitario independiente del personal del programa. En ambos casos se observó que ocurría por dos factores: porque existieron respuestas a demandas concretas, y porque la presidenta era una líder de la comunidad con larga trayectoria, por lo que no dependía de la CNCH para gestionar recursos para su comunidad:

Pues mire, más que nada tenemos que gestionar las cosas para que nos pueda escuchar; para que se nos pueda escuchar. Claro que nos tardamos mucho, porque tenemos que estar metidas, enfocarnos para que, de plano, de plano nos ayuden. Ahorita estamos contentos porque duramos muchos años gestionando la carretera de enseguida; fueron años de gestionarla y pues ya la gente estaba desilusionada: iba yo y me decían: bueno, pues bueno, quieres hacerle la lucha. Y ahorita la gente está muy contenta porque nos están haciendo una avenida muy bonita. Este, fue ahora que empezamos también a través de los comités. (Entrevista personal RS, Ciudad Juárez, 2016)

La presencia de estos factores fue esencial para que funcionaran los Comités Comunitarios de la $\mathrm{CNCH}$ más allá de la tutela del Estado que, al final, es la expectativa de la Política Social de Nueva Generación: que las comunidades se hagan responsables de su propio desarrollo, y que 
los beneficiarios sean ciudadanos activos. Pero como se observó, los elementos de capital social de las comunidades estudiadas se construyeron a partir de las historias de organización de las colonias, existentes antes de la llegada de la $\mathrm{CNCH}$. Se puede concluir entonces que este programa no generó nada nuevo en los términos dados, debido a la falta de continuidad del proceso y de respuesta a las demandas, todo lo cual impidió a las comunidades reconocer que un trabajo reflexivo y sistemático tiene sus frutos a mediano plazo y que una participación, activa y consciente, posibilita el desarrollo social.

\section{Conclusiones}

En Ciudad Juárez existe una experiencia de participación y organización comunitaria muy importante que se ha construido desde la fundación de las colonias, por lo cual se puede hablar de una larga historia de generación de capital social. Sin embargo, esas experiencias se aprovechan particularmente cuando existe un interés en común explícito; lo habitual es que se use esa experiencia para promover a algún candidato de algún partido político. Esta dinámica crea en las comunidades cierta división o conflicto, ya que sienten que los políticos se acercan a ellos únicamente cuando quieren promover a alguien y que en realidad no existe un genuino interés por hacer cambios estructurales en las comunidades. La Cruzada Nacional contra el Hambre ha tratado de recuperar esa organización y fortalecerla a través de los Comités Comunitarios. Se reconoce que la estrategia de promoción de estas figuras de participación tiene un espíritu democrático en su diseño, y que los Diagnósticos Comunitarios utilizados en el desarrollo del programa pueden ser una herramienta que genere reflexión y diálogo para la identificación de los problemas sociales de la comunidad reconocidos por los propios vecinos que los viven diariamente.

El diálogo y la comunicación se dieron en las asambleas que forman parte de la metodología de los Comités Comunitarios: en colectivo se tienen ideas más claras de las necesidades más urgentes que tiene la comunidad. Cabe destacar que eso forma parte del diseño del programa, sin embargo, los y las informantes manifestaban un distanciamiento entre el diagnóstico y el seguimiento. En realidad, se plantea que el programa quedó inconcluso: únicamente se levantó la información de las necesidades, pero no se les atendió, lo que detuvo el proceso participativo que se había iniciadoy generó desconfianza hacia las instituciones y entre los miembros de la comunidad.

Se considera que la estrategia de promover los diagnósticos ciudadanos puede generar un impacto social en las comunidades, pero para ello se necesita dar continuidad a los programas. También es importante señalar que las comunidades tienen posibilidades de auto-organización, la historia de cada colonia revisada así lo demuestra, por lo que no siempre precisan de la ayuda del Estado para generar la organización, a veces sólo falta una pequeña acción para que las comunidades continúen con su trabajo colectivo.

La identificación de necesidades de manera colectiva es un avance importante dentro de las comunidades, y si cuentan con apoyo por parte del Estado es más probable que tengan éxito en el cumplimiento de sus metas. En la actualidad, se discute dentro de los círculos de la academia si las comunidades pueden seleccionar $\mathrm{o}$ identificar las necesidades que empujen su desarrollo. Se habla aquí de atender problemas muy complejos que no tienen una salida sencilla (Cejudo \& Michel, 2015); se requiere de un análisis profundo de la realidad para pensar en pequeñas aristas que posibiliten indicar dónde debe hacerse la intervención social; y quienes mejor conocen esos problemas son aquellos que los viven diariamente: las mujeres y los hombres que conforman una comunidad.

Para que los cambios sociales se realicen en las comunidades se requiere la participación social, voluntad política y 
un trabajo coordinado entre los diferentes órdenes de gobierno, de manera tal que no exista duplicidad de las acciones públicas y se alcance la eficiencia de los recursos. Se pudo observar que las comunidades se organizan y participan porque históricamente lo han hecho y han visto que la organización funciona, pero esto se mantiene cuando se ven logros concretos.

En el caso de la $\mathrm{CNCH}$, la invitación a la primera asamblea para la construcción de los Comités Comunitarios fue favorable, pero la participación fue decayendo conforme no llegaban acciones puntuales que dieran solución a necesidades cotidianas de la comunidad, de manera que se mantuviera vivo el interés en el trabajo colectivo. Si bien es loable una propuesta participativa en la política social como la analizada, el capital social negativo -como la tradicional organización a partir de un partidoy sus promesas de campaña- merma la capacidad de construir beneficiarios activos que reconozcan la importancia de la organización a largo plazo.

Para lograr el alcance de los derechos sociales a través de una política pública se requiere que los funcionarios piensen al ciudadano como alguien reflexivo que debe ser escuchado e invitado al diseño de la política pública, pero para ello se necesita que el Estado rediseñe la forma como se acerca a los ciudadanos. En cuanto a la política social, tendrá que crear un esquema a dos niveles: por una parte, diseñar un proceso participativo con logros a mediano plazo; y por otra, acciones que permitan cubrir las necesidades más inmediatas de aquellos que respondieron a la convocatoria. Por lo tanto, en ocasiones será adecuado desarrollar programas asistenciales, donde el beneficiario es un agente pasivo, pues debe de considerarse que hay individuos que pueden tener una condición crítica que requiere acciones inmediatas y puntuales, por ejemplo, sujetos que tienen alguna enfermedad terminal o comunidades con poblaciones en pobreza alimentaria. Sin embargo, una política de desarrollo social debe diseñarse pensando en abonar a la construcción de la ciudadanía y al alcance de los derechos sociales, reconociendo las diversidades de los territorios, como se hace en la propuesta de los Comités Comunitarios de la $\mathrm{CNCH}$, pero llevando estos planteamientos a la práctica, lo que no se logró en los casos revisados por la falta de respuesta y de continuidad. Se puede afirmar que las comunidades tienen las bases de capital social: confianza y organización social, pero esto no alcanza para transformar su realidad, se precisa del trabajo en conjunto de los diferentes sectores de la sociedad.

En la actualidad, la política social en México se ha planteado como objetivo transformar la condición social y económica de la población que le permitan el alcance de sus derechos sociales, sin embargo, hay estructuras que impiden que este proyecto se realice. Por una parte, existen factores educativos, económicos y sociales, esto es, capital social negativo que no permite la adopción de una nueva forma de relacionarse con el Estado; por otra, falta de continuidad y de respuesta por parte del organismo que dirige la estrategia central de la Política Social de Nueva Generación.

\section{Referencias Bibliográficas}

Álvarez, C. (2015). Capacitación de líderes comunitarios como gestores sociales en Ciudad Juárez, Chihuahua. Estudios Sociales, 45, 263-281.

Arneil, B. (2006). Diverse Communities: The Problem with Social Capital. Cambridge: Cambridge University Press.

Arriagada, I. (2003). Capital social: potencialidades y limitaciones analíticas de un concepto. Estudios Sociológicos, 557-584.

Arzaluz Solano, S. \& Jurado Montelongo, M. A. (2006). Estrategias de combate a la pobreza. El Programa Hábitat en ciudades fronterizas del norte de México: los casos de Nogales, Sonora y Ciudad Juárez, Chihuahua. Región y Sociedad, 18(37), 85-126. 
Bourdieu, P. (1986). The forms of capital. In J. Richardson (Ed.). Handbook of Theory and Research for the Sociology of Education (pp. 241-258). New York: Greenwood.

Cejudo, G. M. \& Michel, C. L. (2015). Resolviendo problemas sociales complejos mediante la integración de políticas. El caso de la Cruzada Nacional contra el Hambre en México. Reforma y Democracia, (63), 35-45.

CEPAL. (2001). Capital social y pobreza. Documento preparado para la Conferencia Regional sobre Capital Social y Pobreza. Santiago de Chile: CEPAL.

Coleman, J. S. (1988). Social Capital in the Creation of Human Capital. American Journal of Sociology, 94, 95-120.

Diario Oficial de la Federación (DOF). (2013). Decreto por el que se establece el Sistema Nacional para la Cruzada contra el Hambre.

Diario Oficial de la Federación (DOF). (2014). Decreto por el que se aprueba el Programa Nacional México sin Hambre 2014-2018.

Galeano Marín, M. E. (2007). Estrategias de Investigación Social Cualitativa. Medellín, Colombia: La Carreta.

Gobierno de la República. (s/f.). Plan Nacional de Desarrollo 2013-2018. Programa Nacional México sin Hambre 2014-2018. Logros 2014. Especial. México: Gobierno de la República. Recuperado de https://www.gob. $\mathrm{mx} / \mathrm{cms} / \mathrm{uploads} / \mathrm{attachment/}$ file/27139/2ope_nacional_ mexicosinhambre.pdf

Jusidman, C. (2008). Introducción. En C. Jusidman Rapoport (Ed.), Reflexiones ciudadanas sobre la política social. México, D.F.: Secretaría de Desarrollo Social, 7-19.

Martínez Flores, V. \& Romo Aguilar, L. (2016). La Cruzada Nacional contra el Hambre en la Frontera Norte: análisis de la implementación en dos ciudades del norte de México. En $5^{\circ}$ Congreso Nacional de Ciencias Sociales. "La Agenda Emergente de las Ciencias Sociales: Conocimiento, crítica $e$ intervención” (1382-1399). México: CUCSH, Universidad de Guadalajara.

Programa Sectorial de Desarrollo Social. (2018). Programa Sectorial de Desarrollo Social 2013-2018. Recuperado de http://www.sedesol. gob.mx/work/models/SEDESOL/ Transparencia/DocumentosOficiales/ Programa_Sectorial_Desarrollo_ Social_2013_2018.pdf

Putnam, R. (1993). Making Democracy Work. Princeton: Princeton Universitiy Press.

Rivera González, J.G. (2016). El deterioro del Capital Social como promotor de la violencia y la delincuencia entre la población del municipio de Rioverde, San Luis Potosí. Papeles de población, $22(87), 103-132$.

Secretaría de Desarrollo Social (Sedesol). (2017). Cruzada Nacional Contra el Hambre. Recuperado de: http:// www.gob.mx/sedesol/acciones-yprogramas/cruzada-nacional-contrael-hambre-18938

Secretaría de Desarrollo Social (Sedesol). (2017). Lineamientos de organización y funcionamiento de los Comités Comunitarios de la Cruzada Contra el Hambre y de los Programa Sociales Federales. Recuperado de http:// www.sedesol.gob.mx/work/models/ SEDESOL/Cruzada/Lineamientos_ COMPLETOS.pdf

Sierra, F. (1998). Función y Sentido de la Entrevista Cualitativa en Investigación Social. En: J. G. Cáceres. Técnicas de Investigación en Sociedad, Cultura y Comunicación. México: Addison Wesley, 277-346. 